\title{
COMPLETE MANIFOLDS OF NONNEGATIVE CURVATURE Marco Zambon
}

\section{Introduction}

The purpose of this survey is to give an overview of the results which characterize Riemannian manifolds with nonnegative or positive sectional, Ricci and scalar curvature, putting an emphasis on the differences between these increasingly strong conditions on curvature. All manifolds considered here are assumed to be complete.

First we consider how nonnegative curvature is different from positive curvature, then we consider how the conditions Scal $\geq 0$, Ric $\geq 0$ and $K \geq 0$ differ from one another. The main part of the survey consists in presenting the most important results concerning manifolds with $K \geq 0$, Ric $\geq 0$ and $S c a l \geq 0$, in particular the topological restrictions that nonnegative curvature implies. We will roughly follow the outline of $[\mathrm{G}]$, a survey-article written by Gromoll in 1991, adding several results, in particular some of those developed after 1991.

The distinction between nonnegative and positive curvature ([G], p.338). In the case of sectional curvature the difference is very subtle. The question, whether $S^{2} \times S^{2}$ (which carries a natural metric with $K \geq 0$ ) admits a metric with $K>0$ is still open (Hopf conjecture). The question is an easier one when we consider complete noncompact manifolds (for if the manifold admits $K>0$ then it must be diffeomorphic to $\mathbb{R}^{n}$ by the Soul Theorem, see Section 4 ), but determining the difference in general between the classes $K \geq 0$ and $K>0$ seems to be an extremely hard problem.

We know more in the cases of Ricci and scalar curvatures. In these cases the difference between positive and nonnegative (although existent) is not too severe. Indeed, Aubin proved that if a manifold has a metric with Ric $\geq 0$ $($ Scal $\geq 0)$ and in a point $p \in M$ Ric $>0(S c a l>0)$, then the metric can be deformed to a metric with Ric $>0(S c a l>0)$ everywhere.

The extent to which the conditions $K \geq 0$, Ric $\geq 0$ and Scal $\geq 0$ are restrictions of each other $([\mathrm{G}]$, p.339). 
It is very difficult to give a general answer to this problem, but there are examples available that provide partial answers. There exist examples of simply connected manifolds of dimension at least 4 which allow Ric $>0$ but not $K \geq 0$, such as connected sums of copies of $S^{l} \times S^{k}$ with $k, l \geq 2$ (metrics with Ric $>0$ can be constructed explicitly, and $K \geq 0$ is ruled out by estimates of the Betti numbers).

There are also examples of manifolds $M$ with $S c a l>0$ which don't admit Ric $\geq 0$ if $\pi_{1}(M)$ is nontrivial, but no examples have been found for simply connected $M$. This is explained by the fact that the main obstruction to $R i c \geq 0$, which is not an obstruction to $S c a l>0$, is the fundamental group of $M$.

The classes $K \geq 0$, Ric $\geq 0$ and Scal $\geq 0$ ([G], p.338 and 348).

The class $K \geq 0$ is very rigid, in the sense that there are tight topological restrictions. Rigidity is even a stronger restriction in the class $K>0$; this explains the scarcity of examples of manifolds with $K>0$ (for $n$ odd and bigger than $24, S^{n}$ is still the only simply connected example known! ([G], p.348)). The methods of the proofs are mainly metric ones (for example Toponogov's Theorem).

In the class $R i c \geq 0$ there are various results concerning the topology of the manifold, but at the same time the topological restrictions are not very tight, so that the amount of examples available is quite large.

The condition $S c a l \geq 0$, on the other hand, doesn't seem to give any general conclusion about the topology or geometry of the manifold.

\section{The class $S c a l \geq 0$}

As mentioned above, the condition $S c a l \geq 0$ doesn't have strong implications, and it has weaker implications for noncompact spaces than for compact ones. We recall (see Section 1) that, if $M$ admits a metric with $S c a l \geq 0$ and $\operatorname{Scal}(p)>0$ at some $p \in M$, then $M$ admits a metric with $S c a l>0$.

It has been shown that, if $M$ is compact and admits $S c a l>0$, then any product with a compact manifold also does. Furthermore, given a compact manifold $M$ with $S c a l>0$, any somewhere positive function on $M$ can be realized as the scalar curvature of a metric conformal to the original one $([\mathrm{G}]$, p.340). It has been also shown that if $M_{1}^{n}$ and $M_{2}^{n}$ are simply connected with $S c a l>0$ and $n \geq 3$, then their connected sum also admits $S c a l>0$ ([G], p.341). 
The main obstruction to $S c a l>0$ for a complete manifold $M$ is given by the fundamental group, and it has been investigated mainly using Spin structures. The concept of enlargeability was introduced to measure how "large" the fundamental group of a manifold is with respect to the manifold. A compact, orientable manifold $M^{n}$ is called an $\varepsilon^{-1}$-hypersurface if there exists a map $f$ of positive degree from $M^{n}$ onto $S^{n}$ such that $d(f(p), f(q)) \leq \varepsilon \cdot d(p, q)$ for all $p, q \in M$ ([GL], p.210). A manifold $M^{n}$ is called enlargeable if for all $\varepsilon>0$ there exists a finite covering which is an $\varepsilon^{-1}$-hypersurface. Enlargeability is a homotopy invariant.

It is a theorem that, if a complete manifold $M$ has a covering space which is an enlargable Spin-manifold, then $M$ can not carry any metric with $S c a l>0$ ([G], p.342). So most manifolds with "large" fundamental group (among them all compact manifold with $K \leq 0$ ) don't admit $S$ cal $>0$. For example the torus $T^{n}$ does not admit $S c a l>0$ if $n \leq 7$, but apparently if $n>7([\mathrm{G}]$, p.342), and any compact 3-manifold with $S c a l>0$ must be a connected sum of copies of $S^{1} \times S^{2}$ and manifolds with finite fundamental group.

\section{The class $R i c \geq 0$}

Quite a lot is known about manifolds with nonnegative or positive Ricci curvature. Manifolds with constant Ricci curvature are called Einstein manifolds, and not very much is known about which obstructions there are for a manifold with Ric $\geq 0$ to be Einstein.

A known fact is Schur's theorem: if $n \geq 3$ and the Ricci curvature is constant at every point of $M$, then $M$ is Einstein ([J], p.132).

Now we will review some classical results for Ric $\geq 0$. Myers' theorem says that, if $M^{n}$ is a complete manifold with Ric $\geq(n-1) C>0$, then $\operatorname{diam}(M) \leq \frac{\pi}{\sqrt{C}}$ and the fundamental group of $M$ is finite. If $M^{n}$ is compact with Ric $\geq 0$, the fundamental group has polynomial growth of order less or equal than $n$, and $b_{1}(M) \leq n$. If $M^{n}$ is complete with Ric $>0$, then $b_{1}(M) \leq n-3$, and if $M^{n}$ is complete with $R i c \geq 0$, any finitely generated subgroup of $\pi_{1}(M)$ has polynomial growth of order less or equal than $n$ ([G], p.351).

So the Ricci curvature sets restrictions on the fundamental group of $M^{n}$, but on the other hand it has been proven that, for any $n \geq 4$, there are compact manifolds $M^{n}$ with Ric $>0$ and arbitrary large homology, as well as com- 
plete manifolds with Ric $>0$ and infinite topological type ([G], p.349; see later in this section for the definition of finite topological type).

An important structural result is the Splitting Theorem ([CG], p.432), which says that if $M$ is complete with Ric $\geq 0$, then $M$ is isometric to $\tilde{M} \times \mathbb{R}^{k}$ where $\mathbb{R}^{k}$ has its standard metric and $\tilde{M}$ contains no lines (lines are geodesics defined for all real numbers, each segment of which is minimal).

Furthermore such an $M$ has at most two ends, and if it has two ends it is isometric to $M^{\prime} \times \mathbb{R}$ where $M^{\prime}$ is compact.

We also have the following result ([CG], p.439): if $M$ is compact with Ric $\geq 0$, the universal cover of $M$ is isometric to some $M_{0} \times \mathbb{R}^{k}$ where $M_{0}$ is a compact simply connected manifold and $\mathbb{R}^{k}$ has its usual metric.

No aspect of the Soul Theorem (see Section 4), which is one of the main structural results for $K \geq 0$, seems to generalize to the case Ric $\geq 0$. Still, the Soul Theorem insipired a result in low dimensions: a noncompact 3-manifold with $R i c>0$ must be diffeomorphic to $\mathbb{R}^{n}([\mathrm{G}]$, p.352). A similar result was proven recently by $\mathrm{Mei}$ and $\mathrm{Xu}$ (see $[\mathrm{MX}]$ ) for noncompact manifolds $M^{n}$ $(n \geq 3)$ with $R i c \geq 0$. Suppose that $\exp _{p}$ is a diffeomorphism for some point $p$ (which is then called a pole), and let $k(s)$ be the maximum of the sectional curvature at points of distance $s$ from the considered pole. If $s^{2} k(s)<\frac{1}{4}$ and $\lim _{s \rightarrow \infty} s^{2} k(s)=0$, then $M^{n}$ is isometric to $\mathbb{R}^{n}$ with the usual metric.

Now we will investigate the implications of Ric $\geq 0$ on the metric of the manifold. The condition Ric $\geq 0$ gives upper bounds for the volumes of metric balls about some $p \in M$ by a theorem of Bishop: if Ric $\geq 0$, a ball $B_{r}(p)$ has less or equal volume than $B_{r}^{n}$, the ball of corresponding radius in flat $\mathbb{R}^{n}$, and the ratio $\frac{\operatorname{Vol}\left(B_{r}(p)\right)}{\operatorname{Vol}\left(B_{r}^{n}\right)}$ is non-increasing ([SH], p.393). This fact can be generalized in the case $K \geq 0$ (see Section 4 ).

We present two recent results related to volume-growth. $M$ is said to have large volume growth if $\alpha_{M}=\lim _{r \rightarrow \infty} \frac{\operatorname{Vol}\left(B_{r}(p)\right)}{\operatorname{Vol}\left(B_{r}^{n}\right)}>0$. This definition is indipendent of the choice of $p \in M$. It was shown by Perelman that there exists a constant $\varepsilon(n)$, depending only on $n$, such that every manifold $M^{n}$ with Ric $\geq 0$ and $\alpha_{M}>1-\varepsilon(n)$ is contractible ([SH], p.393).

A noncompact manifold is said to have finite topological type if there exists a compact subset $N$ of $M$ such that $\partial N$ is a topological manifold and $M \backslash N$ is homeomorphic to $\partial N \times[0, \infty)$. The homology of manifolds of finite topological type is finitely generated. Shen proved that, if $M$ satisfies $R i c \geq 0$, 
$K$ is bounded away from $-\infty, \alpha_{M}>0$ and $\frac{\operatorname{Vol}\left(B_{r}(p)\right)}{\operatorname{Vol}\left(B_{r}^{n}\right)}-\alpha_{M}=o\left(\frac{1}{r^{n-1}}\right)$, then $M$ has finite topological type ([SH], p.394).

If $p, q \in M$ and we define the excess function w.r.t. $p$ and $q$ by

$E_{p, q}(x)=d(x, p)+d(x, q)-d(p, q) \geq 0$, then the condition Ric $\geq 0$ gives an upper bound for $E_{p, q}(x)([\mathrm{G}]$, p.351). This statement is much weaker than Toponogov's Theorem, which holds for $K \geq 0$ (see Section 4).

To close this section we mention Cheng's Sphere Theorem: if Ric $\geq n-1$ and $\operatorname{diam}(M)=\pi$, then $M^{n}$ is isometric to $S^{n}([\mathrm{G}]$, p.352). We recall that, because of Myers' theorem, the condition Ric $\geq n-1$ implies $\operatorname{diam}(M) \leq \pi$.

\section{The class $K \geq 0$}

Spaces of constant curvature have been well understood and classified: if $M^{n}$ has constant curvature $K$, then its universal cover is isometric to the standard sphere with radius $\frac{1}{\sqrt{K}}$ if $K>0$, to flat $\mathbb{R}^{n}$ if $K=0$, or to the hyperbolic space of curvature $K$ if $K<0$ (see [T]). For $n \geq 3$ we have again Schur's Theorem: if $M$ is complete and $K$ is constant at every point, then $M$ has constant curvature ([J], p.132).

Of course, all results which hold for Ric $\geq 0$ also apply to the case $K \geq 0$, and some of them can be improved or completed in the case $K \geq 0$.

Let $M^{n}$ be compact with $K>0$. Synge's Theorem states that, if $n$ is even, then $\pi_{1}(M)$ is trivial if $M$ is orientable and $\mathbb{Z}_{2}$ if $M$ is not orientable. Furthermore, if $n$ is odd, then $M$ is orientable ([G], p.344).

Two theorems were given by Gromov for compact manifolds $M^{n}$ with $K \geq 0$. Firstly ([M], p.21), $\pi_{1}(M)$ is generated by $N \leq \sqrt{2 n \pi} 2^{n-2}$ elements (but this seems to be a very rough estimate). Secondly ([M], p.33), the sum of the Betti numbers of $M^{n}$ w.r.t any field is bounded above by an integer $C(n)$ which depends only on the dimension $n$ of $M$. Again, this estimate seems to be extremely rough, since for all known examples of compact manifolds $M^{n}$ with $K \geq 0$ the sum of the Betti numbers is less or equal than $2^{n}$ (equality is achieved by the torus $T^{n}$ ).

Now we state Toponogov's Theorem for the case $K \geq 0$ (to state Toponogov's theorem it's enough to assume $K \geq C$ for some real $C$; see $[\mathrm{M}]$, p.21). Let $M^{n}$ be a complete manifold with $K \geq 0$, and consider a geodesic triangle with distinct vertices $q, p_{1}, p_{2}$ and edges $c, c_{1}, c_{2}$ such that each $c_{i}$ goes from $q$ to $p_{i}$ and is a minimal geodesic. We denote the angles at $p_{i}$ by 
$\alpha_{i} \in[0, \pi]$. If $L(c) \leq L\left(c_{1}\right)+L\left(c_{2}\right)$, there exists a triangle $\tilde{q}, \tilde{p_{1}}, \tilde{p_{2}}$ in flat $\mathbb{R}^{2}$ with $L(c)=L(\tilde{c}), L\left(c_{i}\right)=L\left(\tilde{c}_{i}\right)$, such that the corresponding angles $\tilde{\alpha}_{i}$ satisfy $\tilde{\alpha}_{i} \leq \alpha_{i}$. This theorem is one of the main methods of proof in the case $K \geq 0$.

The Soul Theorem, due to Cheeger and Gromoll ([M], p.39 and [CG], p.422) is an important structural result, because it essentially reduces the study of manifolds with $K \geq 0$ to the compact case. It is a stronger statement than the fact that every complete noncompact manifold with $K \geq 0$ is homeomorphic to the interior of a compact manifold with boundary, and hence has finitely generated homology ([M], p.33).

The Soul Theorem states the following: if $M$ is a complete noncompact manifold with $K \geq 0$, there exists a compact totally convex, totally geodesic submanifold $S$ of $M$ such that $M$ is diffeomorphic to the normal bundle $\nu S$ of $S$. A submanifold $N$ of $M$ is said to be totally convex if any geodesic segment connecting two points in $N$ has its image in $N$. A submanifold $S$ as above is called a soul and is not uniquely determined, but any two souls are isometric.

In the case $K>0, S$ is a point, so $M$ is diffeomorphic to $\mathbb{R}^{n}$ (and every nonconstant geodesic $\gamma(t)$ goes to infinity as $t \rightarrow \infty$ ([GM], p. 86)).

If $\operatorname{codim}(S)=1$, then $\left.\exp \right|_{\nu S}$ is an isometry between $\nu S$ (with its standard metric) and $M$. In general, we have the following results.

In [GU] it was shown that, given a fixed soul $S$, the metric on $M$ can be deformed to a metric with respect to which $K \geq 0, S$ is still a soul, and a diffeomorphism between $\nu S$ and $M$ is given by $\left.\exp \right|_{\nu S}$. The proof makes use of a map $s h: M \rightarrow S$, which sends a point $x \in M$ to its closest point on $S$, and which turns out to be a $C^{1}$ Riemannian submersion ([TA], p.3036).

In general, $M$ is not even locally isometric to a product $S \times \mathbb{R}^{k}$ (but it is isometric to a product $\tilde{M} \times \mathbb{R}^{k}$, where $\tilde{M}$ contains no lines and $\mathbb{R}^{k}$ has its standard metric, cfr. the Splitting Theorem). In [MA], p. 263 it was shown that, if a soul $S$ of $M$ has trivial normal holonomy, then $M$ is isometric to the product $S \times \mathbb{R}^{k}$, where $\mathbb{R}^{k}$ has nonnegative sectional curvature.

Now we turn our attention to the so-called Sphere Theorems, which are good examples of how manifolds with $K \geq 0$ tend to be rigid. The most classical ([M], p.29) is due to Rauch, Berger and Klingenberg, and says that if $M^{n}$ is simply connected, complete with $4 \geq K>1$, then $M$ is homeomorphic to $S^{n}$. This result is sharp; Berger proved that, if we replace the curvature bounds with $4 \geq K \geq 1$, then $M$ is homeomorphic to $S^{n}$ or isometric to 
complex, quaternional or octavian projective spaces (see $[\mathrm{T}]$ ).

A later version by Grove and Shiohama ([M], p.29) states that if $M^{n}$ is complete with $K \geq 1$ and $\operatorname{diam}(M)>\frac{\pi}{2}, M$ is again homeomorphic to $S^{n}$. The last theorem represented a novelty for two reasons. Firstly, it showed that upper bounds for positive curvature don't affect strongly the topology of manifolds, since they can be replaced by a lower bound of the diameter. Secondly, the proof made use of a new concept of critical point of the distance function, which is defined even where the distance function is not differentiable.

We note that under the assumption of the Grove-Shiohama theorem, we necessarily have $\operatorname{diam}(M) \leq \pi$, and if $\operatorname{diam}(M)=\pi$ then $M$ is isometric to $S^{n}$ by the Cheng's diameter theorem (see Section 3).

We also remark that, if we replace the condition on the curvature in the classical sphere theorem with $1.31 \geq K>1$, then $M^{n}$ is diffeomorphic to $S^{n}$ ([G], p.345).

Positive curvature in a manifold $M$ also has an effect on the injectivity radius $i(M)$, hence also on the diameter: if $M$ is complete, orientable and even dimensional with $K \geq C>0$ (these are also the hypotheses of Synge's theorem), then $i(M) \geq \frac{\pi}{\sqrt{C}}$. Furthermore, if $M$ is complete, simply connected and $C \geq K>\frac{C}{4}>0$ (these are the hypothesis of the sphere theorem), then again $i(M) \geq \frac{\pi}{\sqrt{C}}($ see $[\mathrm{T}])$.

Finally, we consider again the volume of balls. Let $N$ be a compact totally geodesic submanifold of $M$, let $B_{r}(N)$ denote the ball of radius $r$ about $N$, and let $\omega_{k}$ be the volume of the $k$-dimensional unit ball in $\mathbb{R}^{k}$, where $k=$ $\operatorname{codim}(N)$. Then the function $\frac{\operatorname{Vol}\left(B_{r}(N)\right)}{\omega_{k} r^{k}}$ is nonincreasing. From this follows that the volume growth of $M, V G(M)=\inf \left\{x \in \mathbb{R}: \lim _{r \rightarrow \infty} \frac{\operatorname{Vol}\left(B_{r}(p)\right)}{r^{x}}=0\right\}$ (which is independent of $p \in M$ ) is smaller or equal than the codimension of any compact totally geodesic submanifold of $M$ ([TA], p.3035).

\section{Final remarks}

In this paper we tried to point out the main features of manifolds of positive sectional, Ricci and scalar curvature, and we were able to achieve partial results in the classification of such manifolds according to topological features. The Splitting Theorem in the case Ric $\geq 0$ and the Soul Theorem 
in the case $K \geq 0$ are the main results in this sense.

But, as already mentioned, a complete classification seems hopeless, and the fact that the Hopf conjecture ("is there any metric with $K \geq 0$ on the 2Torus?") doesn't yet have an answer confirms this tendency.

If the problem concerning manifolds of non-negative curvature is hard, at leastthe one concerning manifolds with non-positive curvature seems to be simplier: for example it has been proved that any differentiable manifold of dimension 3 or greater admits complete metrics of negative Ricci curvature, so negative Ricci curvature doesn't imply any restriction on the topology of the manifold ([J], p.206).

\section{References}

[CG] Cheeger, Jeff and Gromoll, Detlef; On the structure of Complete Manifolds od Nonnegative Curvature, The Annals of Mathematics, Second Series, Vol. 96, Issue 3 (Nov. 1972), 413-433.

[G] Gromoll, Detlef; Spaces of Nonnegative Curvature, Preceedings of Symposia in Pure Mathematics, Vol 54, Part 3 (1993), 337-356.

[GL] Gromov, M. and Lawson, H.B.; Spin and scalar curvature in the presence of a fundamental group, I, The Annals of Mathematics (2), Vol. 111 (1980), 209-230.

[GM] Gromoll, Detlef and Meyer, Wolfgang; On compete open manifolds of positive curvature, The Annals of Mathematics, Second Series, Vol. 90, Issue 1 (Nov. 1969), 75-90.

[GU] Guijarro, Luis; Improving the metric in an open manifold with nonnegative curvature, Preceedings of the AMS, Vol. 126, Number 5 (1998), 1541-1545.

[J] Jost, Jürgen; Riemannian Geometry and Geometric Analysis, Springer Verlag, second edition (1998).

[M] Meyer, Wolfgang; Toponogov's theorem and applications, lecture notes, College on Differential Geometry, Trieste (1989).

[MA] Marenich, Valery; The Holonomy of Open Manifolds of Nonnegative Curvature, Michigan Math. J. 43 (1996). 
[MX] Mei, Jiaquiang and Xu, Senlin; Almost flat theorem for complete open Riemannian manifolds with nonnegative Ricci curvature, Northeast. Math. J. 14 (1998), no. 3, 305-310.

[SH] Shen, Zhongmin; Complete manifolds with nonnegative Ricci curvature and large volume growth, Inventiones Math 125 (1996), 393-404.

[T] Thorbergsson, Gudlaugur; Vorlesungen ueber Differentialgeometrie, lecture notes of lectures held at the University of Cologne (1998).

[TA] Tapp, Kristopher; Volume growth and holonomy in nonnegative curvature, Preceedings of the AMS, Vol. 127, Number 10 (1999), 3035-3041. 